\title{
Arrays of indefinitely long uniform nanowires and nanotubes
}

\author{
Mecit Yaman ${ }^{1,2}$, Tural Khudiyev ${ }^{1,2}$, Erol Ozgur ${ }^{1,2}$, Mehmet Kanik ${ }^{1,2}$, Ozan Aktas ${ }^{1,3}$, Ekin O. Ozgur ${ }^{1,2}$, \\ Hakan Deniz ${ }^{1,2}$, Enes Korkut ${ }^{1,2}$ and Mehmet Bayindir ${ }^{1,2,3 \star}$
}

\begin{abstract}
Nanowires are arguably the most studied nanomaterial model to make functional devices and arrays ${ }^{1,2}$. Although there is remarkable maturity in the chemical synthesis of complex nanowire structures ${ }^{3,4}$, their integration and interfacing to macro systems with high yields and repeatability ${ }^{5-7}$ still require elaborate aligning, positioning and interfacing and post-synthesis techniques ${ }^{8,9}$. Top-down fabrication methods for nanowire production, such as lithography and electrospinning, have not enjoyed comparable growth. Here we report a new thermal size-reduction process to produce well-ordered, globally oriented, indefinitely long nanowire and nanotube arrays with different materials. The new technique involves iterative co-drawing of hermetically sealed multimaterials in compatible polymer matrices similar to fibre drawing. Globally oriented, endlessly parallel, axially and radially uniform semiconducting and piezoelectric nanowire and nanotube arrays hundreds of metres long, with nanowire diameters less than $15 \mathrm{~nm}$, are obtained. The resulting nanostructures are sealed inside a flexible substrate, facilitating the handling of and electrical contacting to the nanowires. Inexpensive, high-throughput, multimaterial nanowire arrays pave the way for applications including nanowire-based large-area flexible sensor platforms, phase-change memory, nanostructureenhanced photovoltaics, semiconductor nanophotonics, dielectric metamaterials, linear and nonlinear photonics and nanowire-enabled high-performance composites.
\end{abstract}

One-dimensional nanostructures such as nanowires, nanotubes and nanoribbons are continuing to be at the forefront of nanoscience and nanotechnology. Two distinct approaches for the fabrication of these structures are the top-down and bottom-up philosophies ${ }^{10}$. During the past decade, chemical synthesis demonstrated the impressive success of the bottom-up approach in achieving controllable composition and morphology $y^{3,4}$ and prototype functional devices ${ }^{1,2,10}$, but less so for the integration of high-density device assemblies. Post-synthesis assembly techniques, designed for this end, such as electric ${ }^{11}$, magnetic-field-assisted alignment and dielectrophoresis ${ }^{5}$, optical and optoelectronic tweezers ${ }^{12}$, laminar flow in microfluidic channels ${ }^{13}$ and micro contact printing ${ }^{14}$ still do not achieve low cost, high throughput, and good reproducibility with high-precision addressability ${ }^{8}$. For some applications chemical synthesis is simply not a fitting choice; for example, it is still difficult to produce very long aligned nanowires ${ }^{15-17}$, and to obtain nanowires on large area substrates ${ }^{18}$, or polymer substrate nanowire integration ${ }^{19}$, requires separate high-temperature nanowire synthesis and subsequent transformation.

On the top-down side, patterning by lithography is a powerful but costly micro- and nanofabrication tool perfected by the semiconductor industry. However, for one-dimensional nanostructure fabrication, it is not much favoured owing to scientific challenges such as resolution, surface roughness, limited chemical composition and its low throughput ${ }^{10}$. Various hybrid techniques are developed for nanowire fabrication to expand the toolkit, such as using photonic crystal fibres ${ }^{20}$ as microfluidic reactors ${ }^{16}$ to synthesize nanowires (templating) and meniscus-controlled solution evaporation ${ }^{17}$ (growth and winding process) to obtain very long nanowires. A few other successfully fabricated top-to-bottom micro- or nanostructures are the photonic crystal fibre, which consists of microtubular enclaves inside a silica fibre ${ }^{20}$ for optical guiding, thermally drawn composite fibres integrating semiconductors, metals and insulators featuring micro- and nanosizes for advanced functionality ${ }^{21-24}$, and silica nanofibres obtained by tapering ${ }^{25}$. Direct attempts to produce very long metallic ${ }^{26}$ and semiconducting ${ }^{27,28}$ microand nanowires inside viscoelastic matrices using thermal size reduction similar to the Taylor wire process $^{29}$ could not produce ordered structures. Arbitrarily distributed filaments are obtained, making it impossible to identify/address each filament as single nanostructures.

Here we report the first successful fabrication of arrays of millions of ordered indefinitely long nanowires and nanotubes in a flexible polymer fibre. Using a new iterative size-reduction process, semiconducting nanowires and hollow-core piezoelectric polymer nanotubes, with diameters below $15 \mathrm{~nm}$, are obtained as wellordered high-density arrays. The nanostructure sizes are uniform for hundreds of metres along the fibre and radially homogeneous in the cross-section. Unique multimaterial core-shell nanowire arrays were obtained and nanowires and nanotubes can be extracted to obtain extremely long free-standing slivers.

To fabricate nanowires that bridge the macroscopic and the nanoscopic world, we designed a multistep iterative thermal sizereduction process inspired from composite-fibre drawing from polymer reels. We start with a macroscopic polymer rod with an annular hole which is tightly fitted with a thermoelastically compatible material that is to become nanowires on multiple axial elongation and radial reduction. Millimetric fibres obtained from the first thermal size-reduction step are cut and arranged in hexagonal lattices inside a protective jacket, vacuum consolidated and redrawn. This second step reduces the wire size to a few micrometres. The drawing step is repeated a third time with the fibres obtained from the previous step to obtain nanometresized wires (Fig. 1, see also Supplementary Fig. S1). Typically, macroscopic rods are reduced by 25 - to 300 -fold within each step. Using a reduction factor of $50-100$ for three iterative steps, a $10 \mathrm{~mm}$ initial rod is reduced to hierarchically ordered $10 \mathrm{~nm}$

${ }^{1}$ UNAM-National Nanotechnology Research Center, Bilkent University, 06800 Ankara, Turkey, ${ }^{2}$ Institute of Materials Science and Nanotechnology, Bilkent University, 06800 Ankara, Turkey, ${ }^{3}$ Department of Physics, Bilkent University, 06800 Ankara, Turkey. ${ }^{*}$--mail: bayindir@nano.org.tr. 
a

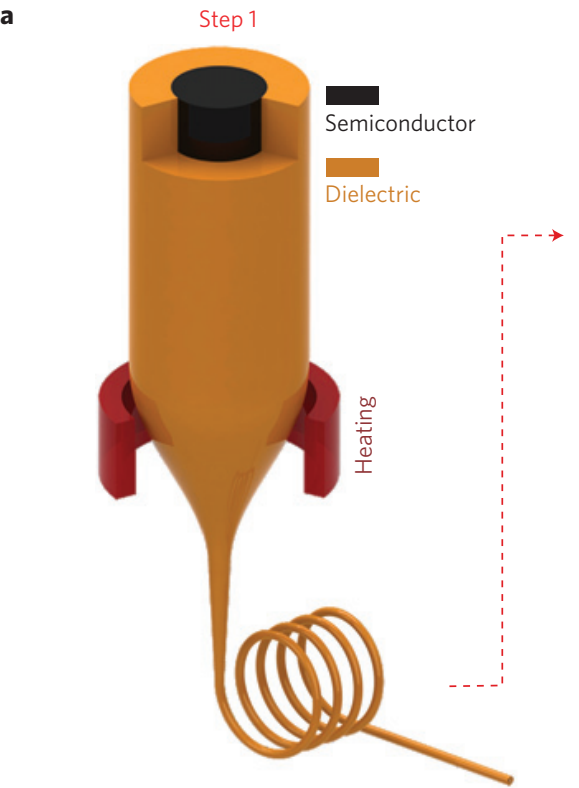

Macroscopic

rod

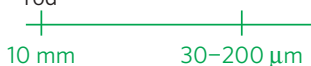

Step 2

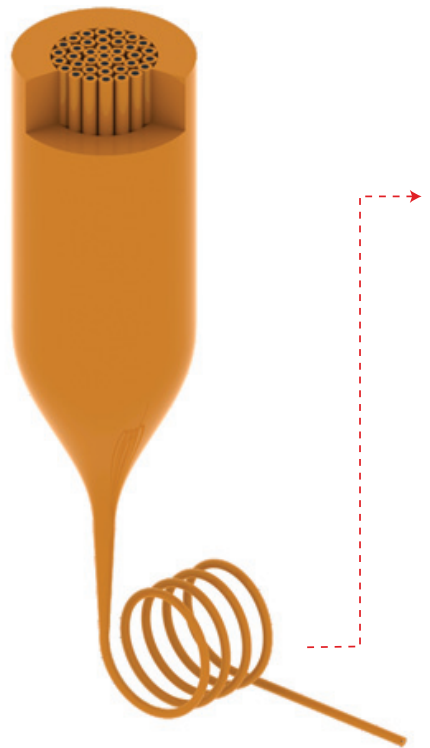

Nanowire array

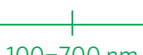

Step 3

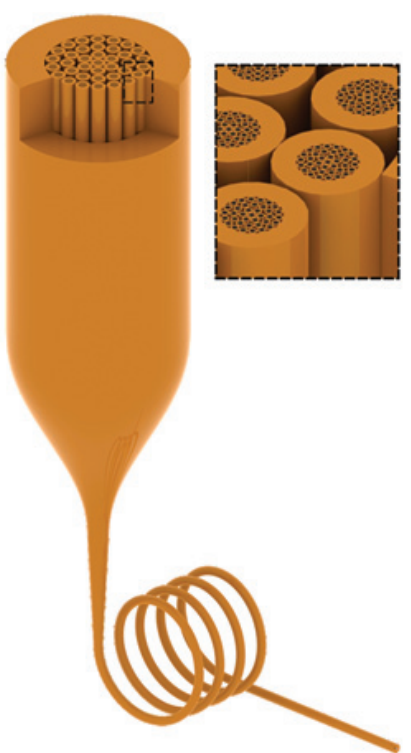

Nanowire array

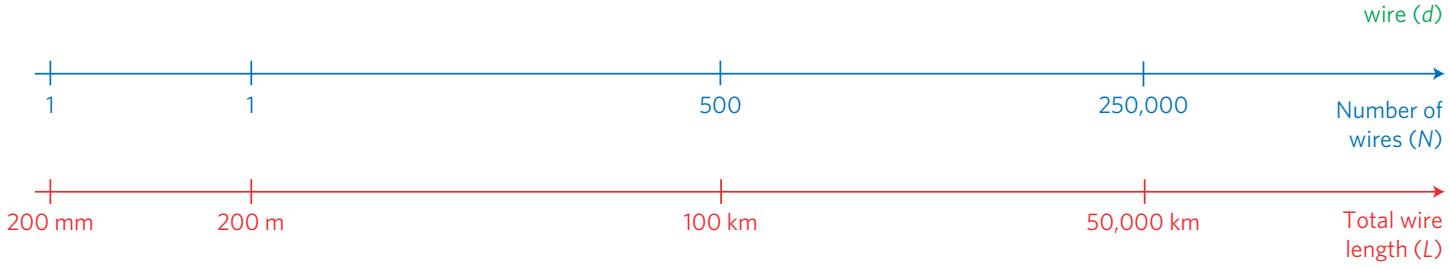

b

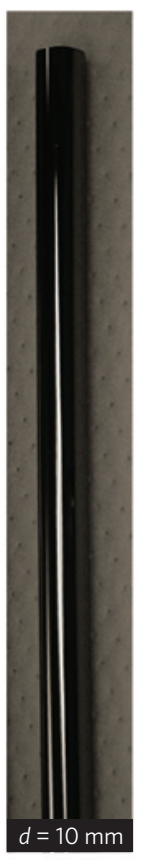

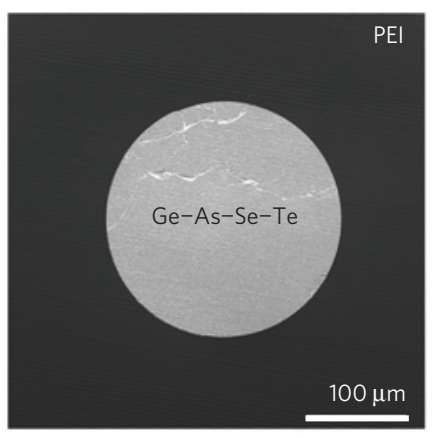

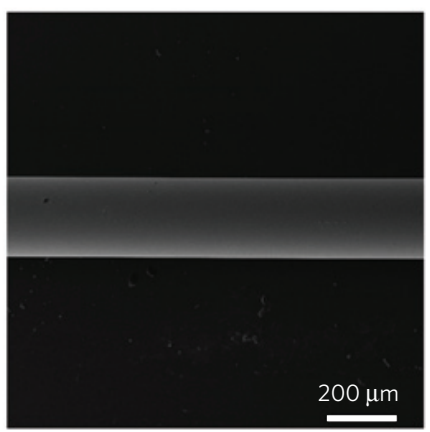

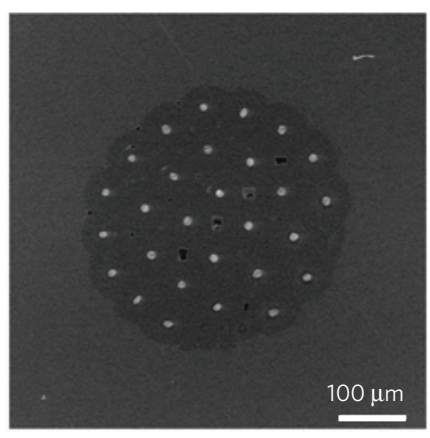

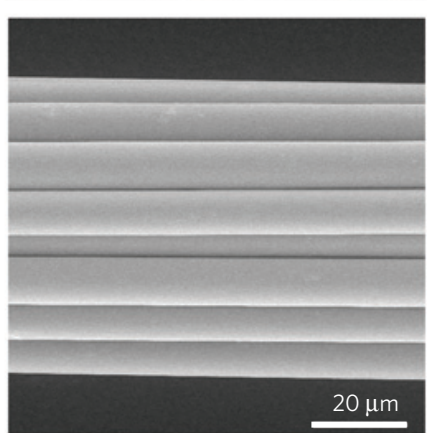

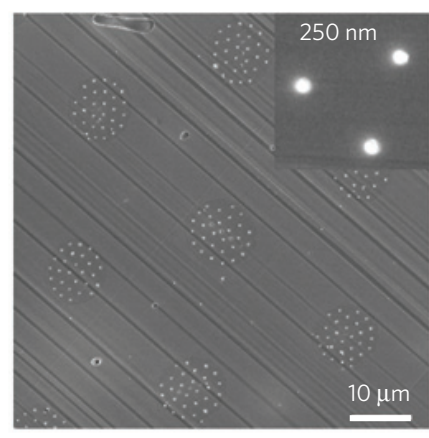

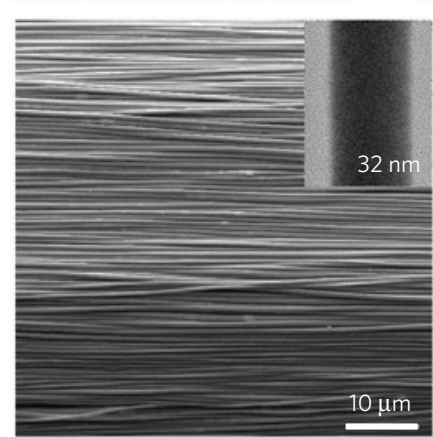

Figure 1 | A new nanofabrication technique, based on iterative size reduction, to produce ordered, indefinitely long nanowire and nanotube arrays. a, A macroscopic multimaterial rod is reduced to ordered arrays of nanowires by thermal size reduction in a protective polymer matrix in successive steps. Each step starts with structures obtained from a previous step, resulting in geometrical size reduction and increment in wire number and length. Using the technique, we produced millions of kilometre-long nanowires with sub-10 nm diameter and an aspect ratio of $10^{11}$. b. As an example, a $10 \mathrm{~mm}$ amorphous semiconducting rod (Ge-As-Se-Te) is reduced to hundreds of metres of single $200 \mu \mathrm{m}$ wire (reduction factor $\times 50$ ), $\sim 30$ wires of $5 \mu \mathrm{m}$ diameter (reduction factor $\times 50$ ) and $\sim 1,000$ wires of $250 \mathrm{~nm}$ (reduction factor $\times 50$ ). Nanowire arrays are obtained as embedded in a dielectric polyetherimide encapsulation. Wire-array cross-sections with well-ordered wires and extracted, globally oriented slivers are shown after each step. Inset: Transmission electron microscopy image of a single 32-nm-thick nanowire obtained by further scaling down the third-step nanowires. 

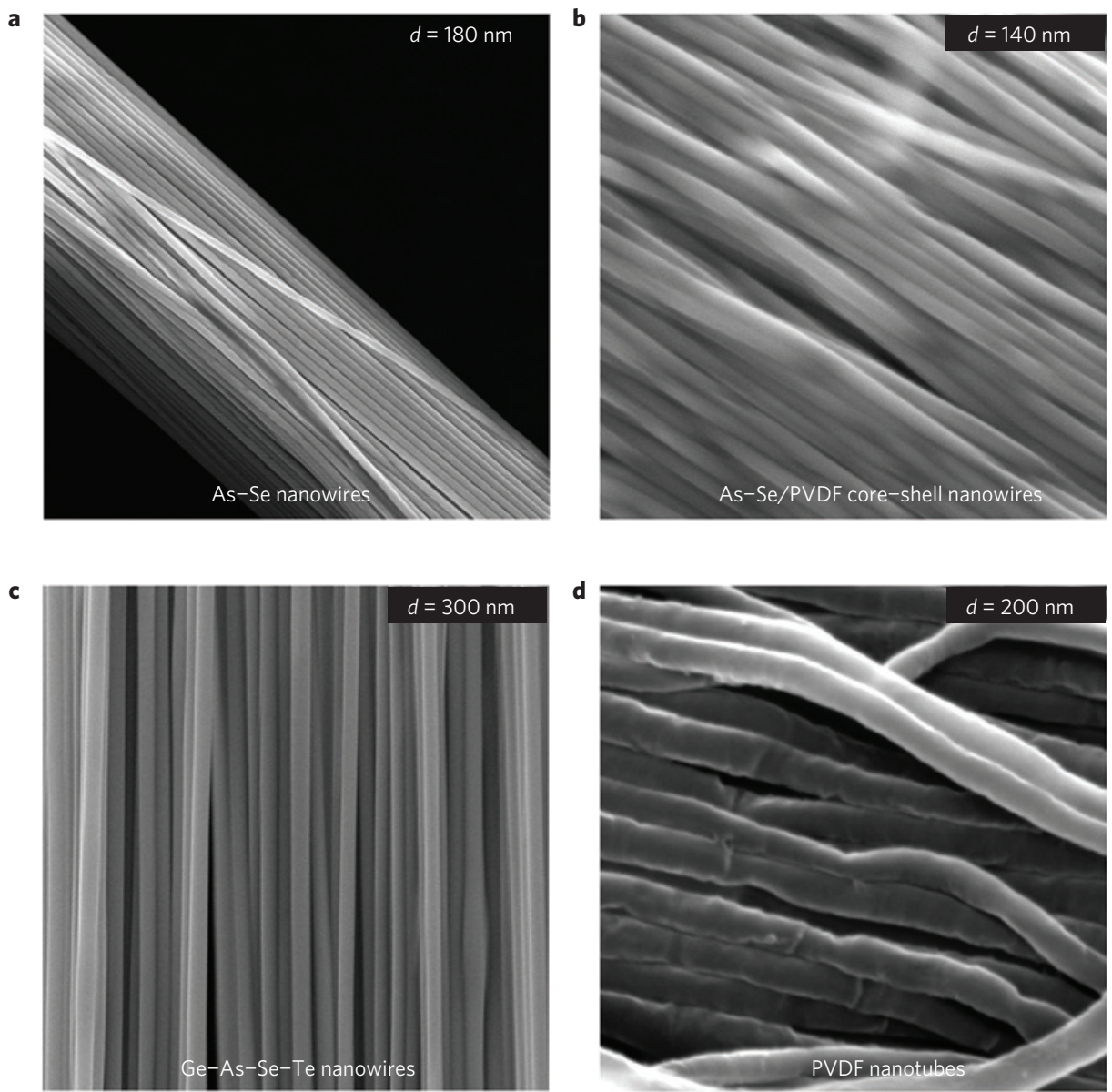

Figure 2 | Globally ordered, multimaterial nanowire, nanotube and cylindrical core-shell arrays. Nanowire and nanotube arrays are extracted from polymer matrix by chemical etching, retaining their global alignment. $\mathbf{a}, \mathrm{As}_{2} \mathrm{Se}_{3}$ semiconducting nanowires. $\mathbf{b}, \mathrm{As}_{2} \mathrm{Se}_{3}$ nanowire core with PVDF encapsulation forming a glass-polymer cylindrical core-shell structure. c, High-refractive-index low-bandgap semiconducting $\mathrm{Ge}_{15} \mathrm{As}_{25} \mathrm{Se}_{15} \mathrm{Te}_{45} \mathrm{nannowire}$ slivers. $\mathbf{d}$, Hollow-core piezoelectric polymer (PVDF) nanotube slivers with $\sim 20 \mathrm{~nm}$ wall thickness. Hollow cores of the tubes are evident from the creases in the slivers after extraction by chemical etching.

wires. The total number of nanowires and their ultimate size distribution is determined by the number of packed fibres after each step, the total number of iterative steps and the reduction factor as shown in Fig. 1.

We demonstrate nanowire production using chalcogenide semiconducting glasses in Fig. 1. Stable glass-making non-oxide chalcogen compounds have low softening points, making them amenable for thermal co-drawing inside polymer matrices owing to their excellent match of thermomechanical properties (Supplementary Fig. S2). We synthesized a 10-mm-diameter glass-making chalcogenide $\mathrm{Ge}_{15} \mathrm{As}_{25} \mathrm{Se}_{15} \mathrm{Te}_{45}$ (GAST, $T_{\mathrm{g}} \sim 190^{\circ} \mathrm{C}$ ) from high-purity elemental constituents using the conventional melt-quenching technique $^{30}$ (see the Methods section). Around the glass rod, a high-temperature engineering polymer (polyetherimide, $T_{\mathrm{g}} \sim$ $220^{\circ} \mathrm{C}$ ) is rolled and thermally consolidated in vacuum. The composite structure is then thermally drawn to reduce radial size (Supplementary Fig. S3). Typically, the process is carried out at $50 \mathrm{~cm} \mathrm{m^{-1 }}$ drawing speed while feeding the macroscopic rod at $1.5 \mathrm{~mm} \mathrm{~min}^{-1}$ into a furnace at $275^{\circ} \mathrm{C}$. The 'fibre wires' obtained from the first drawing are then packed and vacuum consolidated to prepare a second-step macroscopic rod. Vacuum consolidation ensures structural homogeneity and purity from defects that may adversely affect the subsequent thermal drawing process (see the Methods section). Beginning with a $10-\mathrm{mm}$-diameter GAST rod, the diameter is successively reduced to $200 \mu \mathrm{m}, 5 \mu \mathrm{m}$ and $250 \mathrm{~nm}$ within each drawing step. Further reduction in the third step results in sub-50 $\mathrm{nm}$ nanowire arrays. In Fig. 1, we show a perfectly ordered array of GAST micro- and nanowires in the polyetherimide polymer cross-section. It is remarkable that the macroscopic structure is undistorted after the drawing steps. We have also demonstrated that the micro- and nanowires can be extracted from the polymer matrix retaining their global alignment simply by exposing the polymer section to organic etching agents (dichloromethane (DCM) or dimethylacetamide; see the Methods section). Resulting free-standing micro- and nanowire slivers are shown in Fig. 1.

We fabricated a variety of multimaterial nanowire and nanotube structures by using the same technique (Fig. 2; Supplementary Figs S4-S7). Regular nanowire arrays are fabricated from the highly nonlinear glass $\mathrm{As}_{2} \mathrm{Se}_{3}$ (Fig. 2a), ordered core-shell arrays of $\mathrm{As}_{2} \mathrm{Se}_{3}-\mathrm{PVDF}$ (polyvinylidene fluoride; Fig. 2b), high-refractiveindex GAST glass (Fig. 2c), and photoconductive and phasechange Se glass (Supplementary Figs S4-S6). Separately, nanotubes of piezoelectric PVDF (melting temperature $T_{\mathrm{m}}=171{ }^{\circ} \mathrm{C}$ ) are obtained using the same process by using PVDF sheets as the filling material and polysulphone (PSU, $T_{g} \sim 190^{\circ} \mathrm{C}$ ) as the jacket layer (Fig. 2d). PVDF sheets take their tubular shape through melting at the drawing temperature $\left(T_{\text {draw }}=240^{\circ} \mathrm{C}\right)$ followed by self-organization due to high surface energy between the fluoride polymer and the protective PSU jacket. Macroscopic PVDF tubes can be seen after the first and second step drawings, and more interestingly tubular shapes are conserved in the 
a
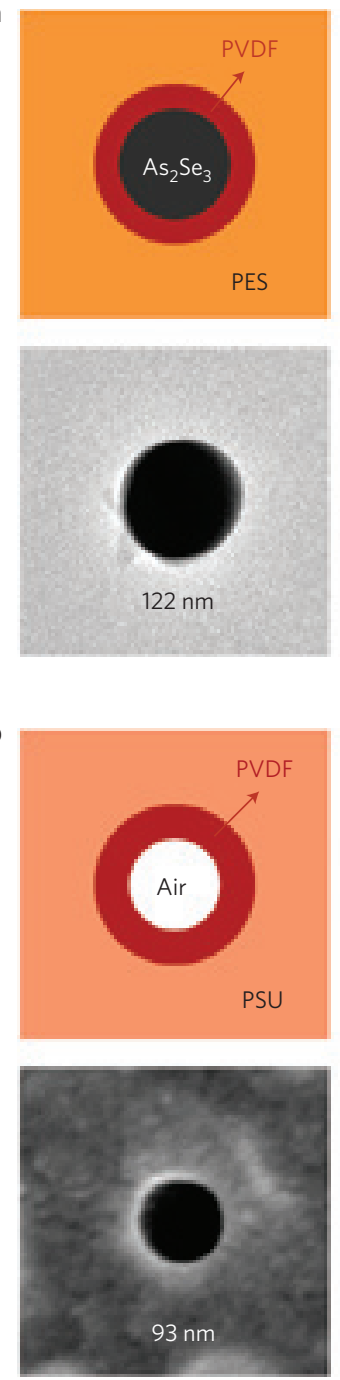
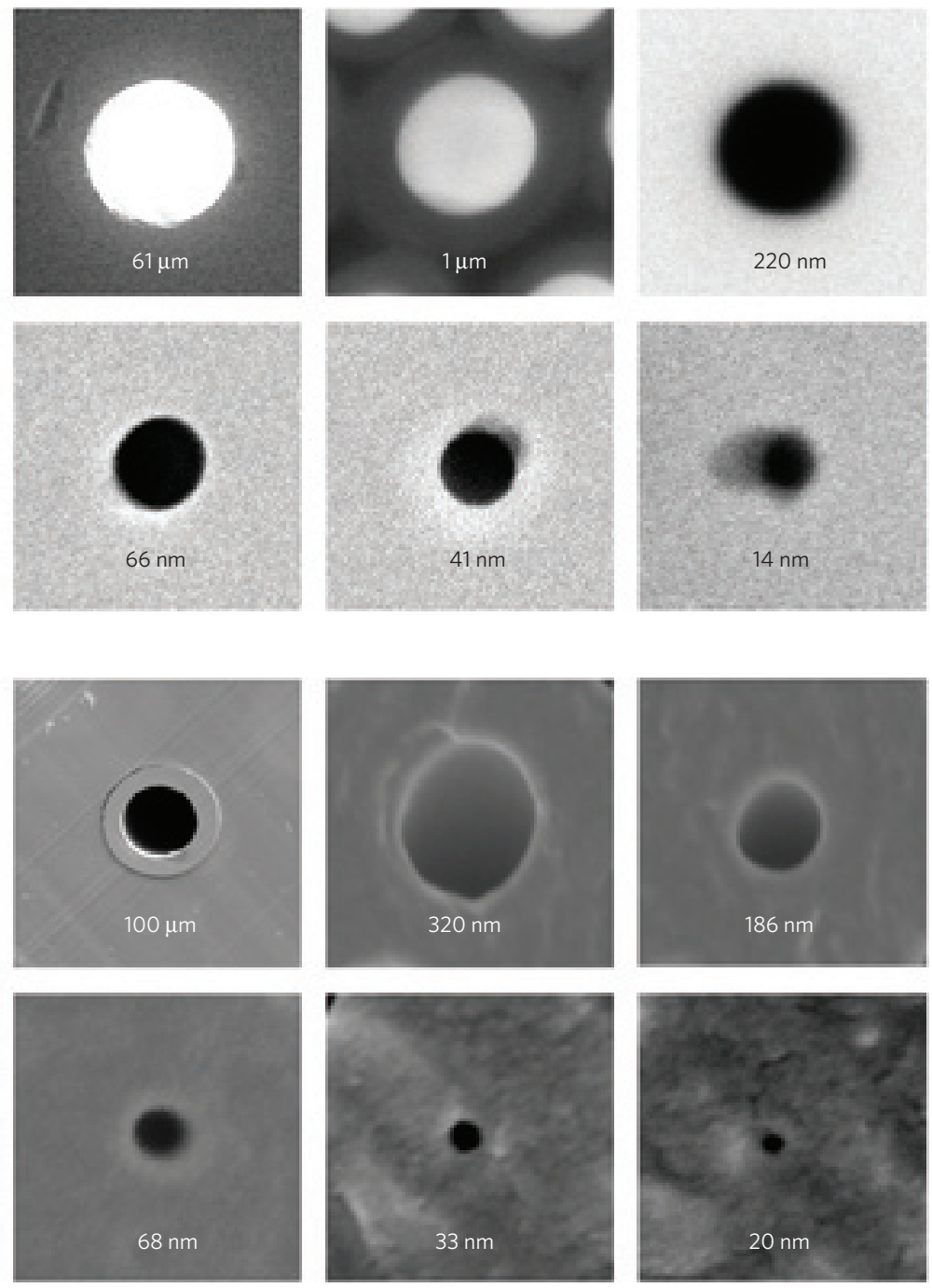

Figure 3 | Regular size reduction and ultimate achievable limit with multimaterial iterative size-reduction technique. We produced kilometre-long nanowires and nanotubes with any diameters within the $100 \mu \mathrm{m}-10 \mathrm{~nm}$ range by preserving ordered geometry. $\mathbf{a}$, $\mathrm{As}_{2} \mathrm{Se}_{3}-\mathrm{PVDF}$ core-shell nanowires scaled regularly from $200 \mu \mathrm{m}$ to $14 \mathrm{~nm}$. b. Hollow-core PVDF nanotubes scaled regularly from $200 \mu \mathrm{m}$ to $20 \mathrm{~nm}$ without losing structural integrity. Smearing around the nanowires is caused during sample preparation with ultramicrotomy.

nanoscale, resulting in very long arrays of nanofluidic channels in a flexible polymer fibre. Thermal melt-drawing of globally aligned PVDF nanotubes is an interesting alternative to existing templating and sol-gel methods for producing high-density nanotube channels ${ }^{31}$.

Nanowire fabrication with low-melting-temperature metals and metal alloys ( $\mathrm{Sn}, \mathrm{SnPb}, \mathrm{SnAg}$ ) was also explored using the same process. Fibre drawing is ideally designed for glassy materials; however, if it is possible to melt the crystalline substance without oxidizing inside a protective glassy matrix during thermal draw, crystalline rods will melt and recrystallize as elongated wires conserving the structural integrity ${ }^{21,22,30}$. Recently, it was shown that other phases (such as metals and semimetals) can be drawn in an encapsulating glassy matrix ${ }^{32}$. Here, drawing occurs in a regime dominated by viscous forces, allowing for internal low-viscosity metallic domains to be arranged in cross-sections confined by viscous glassy boundary layers. However, this latter type of composite drawing becomes especially challenging with submicrometre filament sizes. The Taylor process, other attempts and the authors' own previous work suggests that crystalline materials can be co-drawn in glassy matrices when the drawing conditions are just right. To demonstrate that it is indeed feasible to fabricate ordered arrays of micrometre- and sub-micrometre-scale metallic wires in polymer fibres, we selected tin and tin alloys owing to their low melting temperature $\left(T_{\mathrm{m}}=223^{\circ} \mathrm{C}\right)$. The drawing process is carried out with similar parameters to those of chalcogenide glasses. We started with a 1.6-mm-diameter soldering alloy $\mathrm{Sn}_{96.5} \mathrm{Ag}_{3.5}$ tightly fitted inside a hollow-core polyethersulphone rod, and scaled successively to 60 and $4 \mu \mathrm{m}$ (Supplementary Fig. S7).

We demonstrate the utility of the top-down size-reduction technique by producing wires with sizes spanning the whole microand nanoscale (Fig. 3). Beginning with the same macroscopic rod, structures of six orders of magnitude in size can be obtained. We demonstrate this facility with core-shell $\mathrm{As}_{2} \mathrm{Se}_{3}-\mathrm{PVDF}$ nanowires and hollow-core PVDF tubes. $\mathrm{As}_{2} \mathrm{Se}_{3}-\mathrm{PVDF}$ core-shell nanowires were regularly scaled down to $61 \mu \mathrm{m}, 1 \mu \mathrm{m}, 220,122,66,41$ and $14 \mathrm{~nm}$ (Fig. 3a). Hollow-core PVDF nanotubes were scaled regularly, conserving their structural integrity, from $100 \mu \mathrm{m}$ down to $20 \mathrm{~nm}$ (Fig. 3b). It is remarkable that we produce extended lengths of micro- and nanotubes that are highly sought materials in micro- and nanofluidics research. The wires and tubes are obtained as ordered arrays in the surrounding polymer matrix. In the micrographs, smearing around the nanowires is caused during 

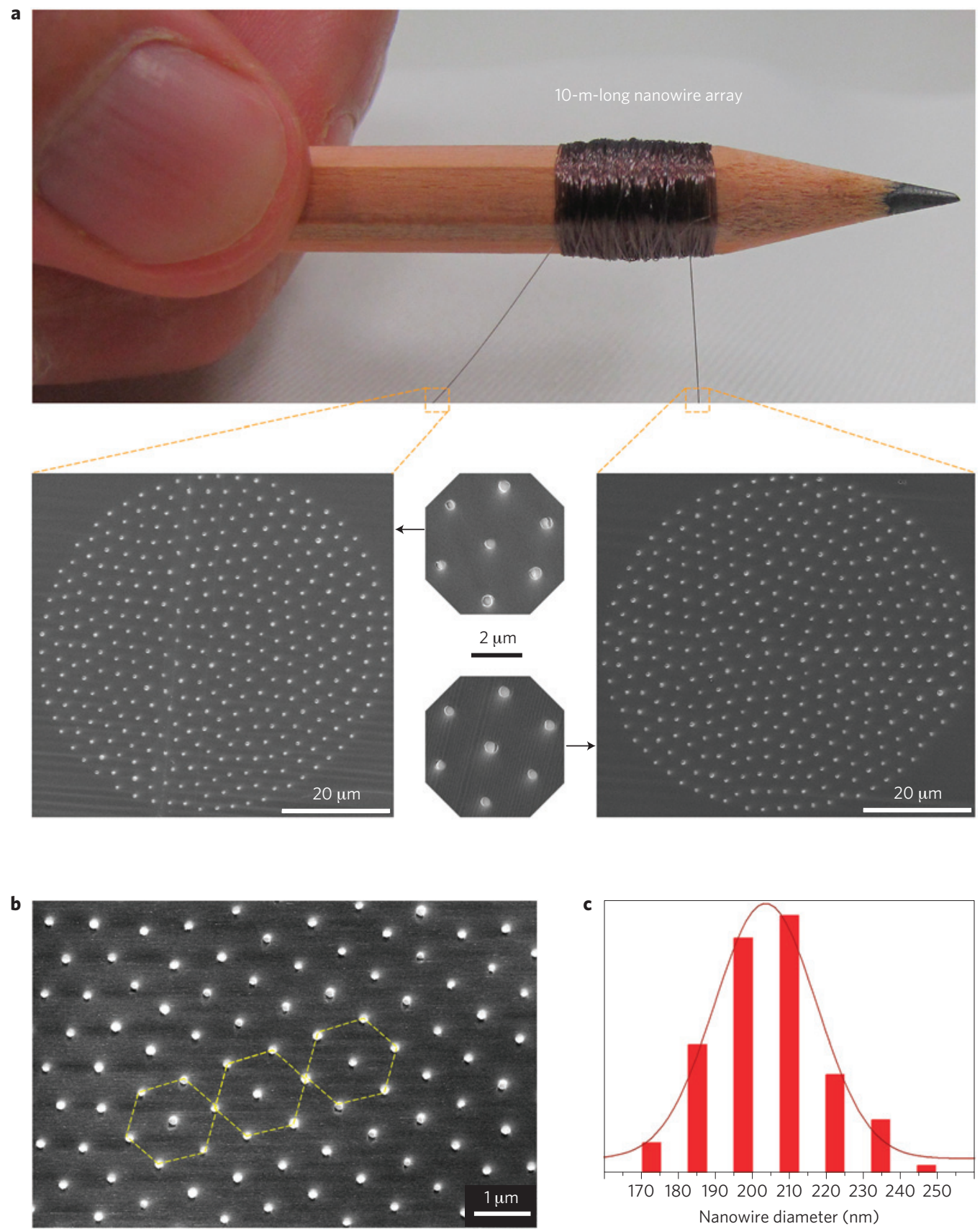

Figure 4 | Radial and axial uniformity of the nanowire arrays. a, A polymer-embedded nanowire array rolled around a pencil truly spans macroscopic and nanoscale worlds. Cross-sectional SEM micrographs from both sides of a 10-m-long polymer fibre that contains hundreds of $\mathrm{As}_{2} \mathrm{Se}_{3}-\mathrm{PVDF}$ core-shell nanowires prove that nanowire arrays are axially uniform to less than $1 \%$ for macroscopic distances. $\mathbf{b}$, High-precision hexagonal packing of core-shell nanowires in the polymer matrix. c, Radial size distribution of the nanowires, shown as a histogram, is uniform with a standard deviation of $6.5 \%$.

sample preparation with microtomy, making it challenging to image embedded nanowires in the polymer matrix. For glassy filling materials, the ultimate size reduction limit depends on the drawing dynamics and viscoelastic properties of the matrix. Amorphous glasses and polymers that soften during drawing can be easily reduced to $10 \mathrm{~nm}$ radial size without axial breakdown. We infer that, with feed-in and draw speeds tuned and controlled carefully, the drawing process can yield molecular wires; the ultimate size reduction limit is still an enticing question.

We demonstrated radial and axial uniformity of the $\mathrm{As}_{2} \mathrm{Se}_{3}-$ PVDF core-shell nanowires by taking cross-sectional scanning electron microscopy (SEM) images (FEI, Nova NanoSEM 600) from both sides of a 10-m-long nanowire-embedded fibre, rolled conveniently on a pencil (Fig. 4). It is extraordinary that the conformal surface coverage of a macroscopic object with nanowires can be achieved trivially. Roughly 400 of the $\mathrm{As}_{2} \mathrm{Se}_{3}-\mathrm{PVDF}$ core-shell nanowires with $200 \mathrm{~nm}$ diameter are obtained with two-step iterative drawing. The two-dimensional array structure and integrity of the embedded nanowire arrays are retained for macroscopic lengths. The size distribution along the fibre length is uniform within $1 \%$ (Fig. 4a). High-precision hexagonal packing of core-shell nanowires in the matrix is shown in Fig. $4 \mathrm{~b}$. The size distribution of the nanowires from the second step is shown as a histogram and found to be uniform within $6.5 \%$ (Fig. 4c).

Finally, we investigated the size-dependent photoconductivity of selenium micro- and nanowire arrays fabricated using the described 
a
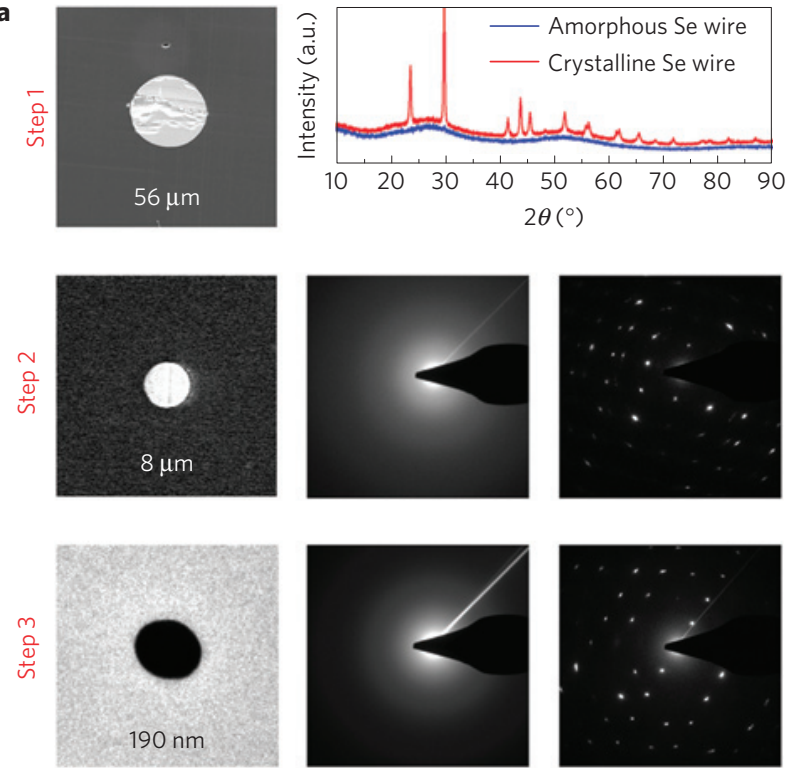

b

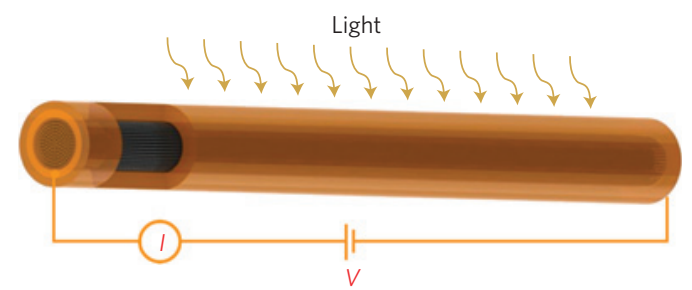

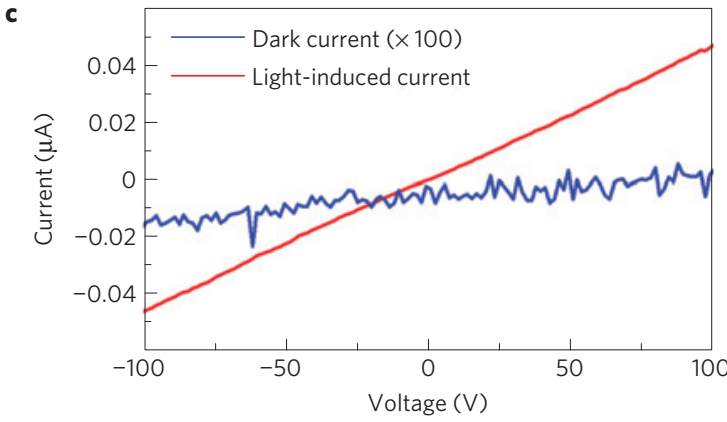

d

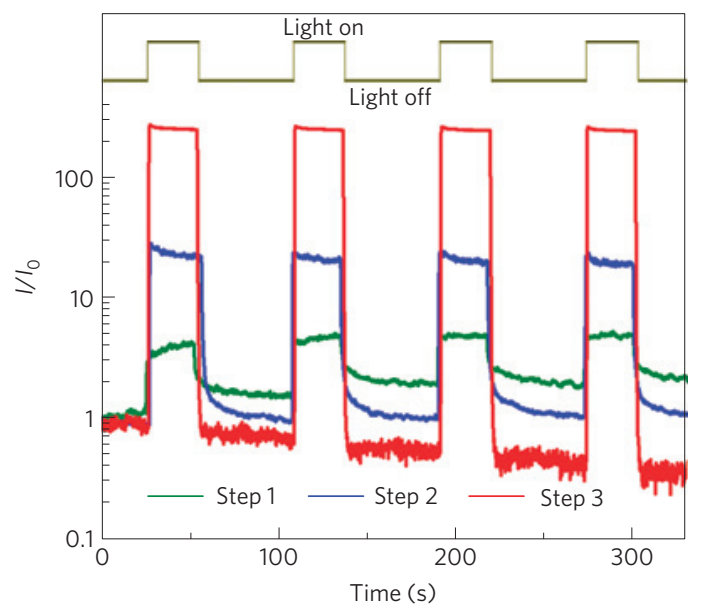

Figure 5 | Photoconductivity of selenium microwire and nanowire arrays. a, Amorphous selenium wires are crystallized through heat treatment or by exposing to the organic reagent pyridine. SEM images of regularly scaled-down individual selenium wires. The electrically conductive hexagonal crystallographic phase is obtained by X-ray and electron diffraction. $\mathbf{b}$, A schematic representation of the photoconductance measurement geometry.

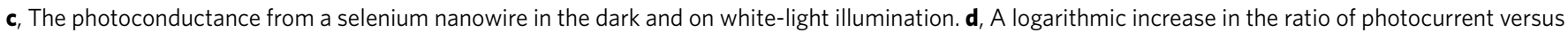

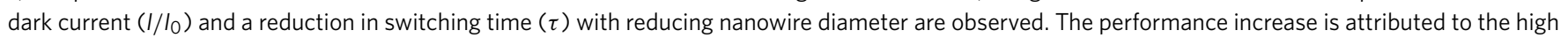
optical density of selenium, increased surface-area-to-volume ratio and enhanced scattering in the polymer matrix.

technique. Elemental selenium has phase-dependent electrical conductivity and photoconductivity properties, making it suitable for phase-change memory, optoelectronics and photodetection applications. In a three-step drawing process, we obtained a $56 \mu \mathrm{m}$ wire, an array of $100 \times 8 \mu \mathrm{m}$ wires and an array of $10,000 \times$ $190 \mathrm{~nm}$ nanowires. The structure of the nanowires inside the polyethersulphone polymer matrix is conserved after the drawing steps (Fig. 5). The uniformities of the selenium nanowires from the second and third steps are found to be $\pm 3 \%$ and $\pm 11 \%$ respectively (Supplementary Fig. S5). Factors contributing to the overall size distribution are slight variations in the fibres that are used to make the macroscopic composite, and the total accumulated size variation from each previous step. Amorphous selenium wires are obtained after drawing, as revealed by X-ray diffraction and transmission electron microscope diffraction (FEI, Tecnai G2 F30). The wires are crystallized through heat treatment, by annealing above the crystallization $\left(T_{\mathrm{x}}=110^{\circ} \mathrm{C}\right)$ but below the melting temperature ${ }^{27,33}$ and by exposing to the organic reagent pyridine (Fig. 5a, see Methods section). After crystallization we observe that the conductivity increases to $10^{-4} \mathrm{~S} \mathrm{~cm}^{-1}$ consistent with bulk selenium conductivities ${ }^{34}$. Electrical measurements were carried out with nanowire arrays by cleaving fibre ends with a razor blade and electrical contacting was achieved using silver paint on the facets of the fibre. Step 3 nanowires were chemically crystallized after extraction from the polymer matrix. Electrical gain due to photoconductivity is measured by shining white light $(50 \mathrm{~W})$ while applying voltage bias (Fig. $5 \mathrm{~b}$ ). Step-3 wires make Ohmic contact and feature photoconductivity after crystallization (Fig. 5c). The photoconductance of the nanowires is observed to be a function of the nanowire number and size in the array. We observed that the $I / I_{0}$ gain (photocurrent versus dark current) was an order of magnitude larger for step-2 wires and two orders of magnitude larger for step-3 wires with respect to the single selenium microwire from the first drawing (Fig. 5d). The switching between on/off states is also faster with the smaller nanowire sizes. We attribute the logarithmic increase in photoconductance to the high absorption of selenium, increased surface-area-to-volume ratio and enhanced scattering of the incoming beam.

We have demonstrated a unique, high-throughput, lithographyfree nanofabrication method to produce macroscopically large nanowire and nanotube arrays. The nanostructures are indefinitely long, ordered, uniform, high-density arrays embedded in a flexible polymer fibre matrix. Diverse multimaterial sets and disparate combinations are used to obtain crystalline semiconducting nanowire arrays, chalcogenide nanowire array waveguides, piezoelectric polymer nanotubes and cylindrical core-shell structures. It is an open question if molecular wires can be obtained using the method. The ultimate size reduction for the nanowires needs to be studied by optimizing drawing parameters for thermoviscous materials and materials that melt during drawing separately. On the other hand, the material set can also be widened to include semiconductors, such as $\mathrm{Si}$ and $\mathrm{Ge}$, that soften/melt at higher temperatures using suitable glassy matrices.

We expect that a whole new family of radically novel nanowire applications will be enabled owing to the unique 
geometry and material set used. We identify a number of enticing applications that are of current and rigorous scientific and technical interest. For example, these nanostructures can be exploited in nanowire electronics, as large-area conformal photodetectors, large-area flexible nanowire sensors ${ }^{15}$, scalable high-density nanowire-based phase-change memory ${ }^{35,36}$ and in high-speed reconfigurable field-effect transistors ${ }^{33}$; in energy harvesting, large-area cylindrical heterostructure nanowires can be used as active cells ${ }^{37,38}$ but also for passive light enhancement in resonanceenhanced third-generation photovoltaics ${ }^{39,40}$; in semiconductor nanophotonics, polymer-embedded chalcogenide nanowires can be used as high-refractive-index dielectric structures for sizedependent absorption ${ }^{41}$, structural colouring ${ }^{42}$ and biomimicry, and as dielectric metamaterials ${ }^{43}$; in nonlinear photonics, ordered nanowires can be used as high-power zero-dispersion optical arrays for new frequency generation ${ }^{44}$ and in discrete optics ${ }^{45}$; in mechanics, nanowire-embedded high-strength $\operatorname{composites}^{29}$; in acoustics, flexible piezoelectric nanowires and nanotubes can be used as sensors and actuators ${ }^{46}$, as pumps, in energy harvesting ${ }^{47}$ and as nanochannels in nanofluidics.

\section{Methods}

Synthesis of chalcogenide-glass rods. $\mathrm{As}_{2} \mathrm{Se}_{3}$ and Se rods, $10 \mathrm{~mm}$ in diameter and $15 \mathrm{~cm}$ in length, are prepared by melting commercially bought glasses (Amorphous Materials) and pellets (Alfa Aesar) in a vacuum-sealed quartz tube followed by water quenching. $\mathrm{Ge}_{15} \mathrm{As}_{25} \mathrm{Se}_{15} \mathrm{Te}_{45}$ glass rods (the same diameter) are prepared from high-purity elements (Alfa Aesar) using the sealed-ampoule melt-quenching technique. The weighted materials are placed in a quartz tube under nitrogen atmosphere in a glove box $\left(\mathrm{H}_{2} \mathrm{O}, \mathrm{O}_{2}\right.$ below $\left.0.1 \mathrm{ppm}\right)$. The quartz tube is kept at $330^{\circ} \mathrm{C}$ for an hour under vacuum to remove surface oxides and sealed $\left(10^{-6}\right.$ torr $)$. The sealed ampoule is then heated to $950^{\circ} \mathrm{C}$, rocked for $18 \mathrm{~h}$ and then quenched in iced water.

Macroscopic-composite preparation, consolidation and thermal size reduction. Macroscopic rods that will undergo axial elongation and radial size reduction are structures approximately $30 \mathrm{~mm}$ in diameter. These structures are prepared in a clean pressure flow room, by tightly rolling polyethersulphone or polyetherimide films around chalcogenide glass rods $(10 \mathrm{~mm}$ diameter) until the diameter is $30 \mathrm{~mm}$ (see Supplementary Fig. S1). Then, the macroscopic structure is thermally consolidated under vacuum $\left(10^{-3}\right.$ torr $)$, above the glass transition temperature of the polymer and glasses to fuse $\left(260^{\circ} \mathrm{C}\right.$ for $\left.30 \mathrm{~min}\right)$. Step- 2 and higher-step structures are prepared with previously obtained polymer-wire fibres and a hollow-core polymer rod fabricated by rolling polymer sheets on a Teflon rod, consolidation and removing the Teflon rod. About 100 fibres with $0.5 \mathrm{~mm}$ diameters are cut as $10 \mathrm{~cm}$ fibres, tightly packed and placed inside the hollow-core polymer rod. The structure is consolidated once more to fuse packed fibres to the outer polymer jacket $\left(250^{\circ} \mathrm{C}\right.$ for $\left.15 \mathrm{~min}\right)$. The second consolidation step ensures structural integrity during drawing. The macroscopic structure is heated and drawn into hundreds of metres of micro- and nanowire in a vertical two-zone furnace, with a top-zone temperature of $275^{\circ} \mathrm{C}$ and bottom zone $200^{\circ} \mathrm{C}$ (see Supplementary Fig. S3). The nanowire diameter is controlled using a laser micrometer by monitoring the fibre diameter.

Nanowire and nanotube extraction. The polymer encapsulating the nanowires and nanotubes was extracted for electron microscopy imaging in the longitudinal dimension by DCM (Carlo Erba). Nanowire or nanotube arrays embedded in polymer were fixed on a glass substrate by means of aluminium foil, and then half soaked in bottles containing DCM. The extracted nanostructures were further rinsed gently with DCM to remove residual polymer. A final $\mathrm{O}_{2}$ plasma treatment (Nanoplas DSB6000) for several hours was applied for chalcogenide and metal nanowires to remove organic residues.

Nanowire crystallization by thermal and chemical methods. Step- 1 selenium nanowires were crystallized by annealing at $150^{\circ} \mathrm{C}$ for $1 \mathrm{~h}$. Step- 2 nanowire crystallization occurred after keeping nanowire arrays at $220^{\circ} \mathrm{C}$ for $15 \mathrm{~min}$ and cooling to room temperature in $1.5 \mathrm{~h}$. For crystallization of step- 3 selenium nanowire arrays, extracted nanowire arrays were soaked overnight in a $10 \%$ pyridine (Riedel-de Haën) solution in isopropanol.

Received 8 January 2011; accepted 28 April 2011; published online 12 June 2011; corrected after print 19 July 2011

\section{References}

1. Lu, W. \& Lieber, C. M. Nanoelectronics from the bottom up. Nature Mater. 6 , 841-850 (2007).

2. Thelander, C. et al. Nanowire-based one-dimensional electronics. Mater. Today 9, 28-35 (October, 2006).
3. Tian, B., Xie, P., Kempa, T. J., Bell, D. C. \& Lieber, C. M. Single crystalline kinked semiconductor nanowire superstructures. Nature Nanotech. 4, 824-829 (2009).

4. Caroff, P., Dick, K. A., Johansson, J., Messing, M. E., Deppert, K. \& Samuelson, L. Controlled polytypic and twin-plane superlattices in III-V nanowires. Nature Nanotech. 4, 50-55 (2009).

5. Li, Y., Qian, F., Xiang, J. \& Lieber, C. M. Nanowire electronic and optoelectronic devices. Mater. Today 9, 18-27 (October, 2006).

6. Freer, E. M. et al. High-yield self-limiting single-nanowire assembly with dielectrophoresis. Nature Nanotech. 5, 525-530 (2010).

7. Takei, K. et al. Nanowire active matrix circuitry for low-voltage macro-scale artificial skin. Nature Mater. 9, 821-826 (2010).

8. Yan, R., Gargas, D. \& Yang, P. Nanowire photonics. Nature Photon. 3, 569-576 (2009).

9. Yang, P., Yan, R. \& Fardy, M. Semiconductor nanowire: What's next? Nano Lett. 10, 1529-1536 (2010).

10. Lieber, C. M. \& Wang, Z. H. Functional nanowires. MRS Bull. 32, 99-104 (2007).

11. Smith, P. A. et al. Electric-field assisted assembly and alignment of metallic nanowires. Appl. Phys. Lett. 77, 1399-1401 (2000).

12. Jamshidi, A. et al. Dynamic manipulation and separation of individual semiconducting and metallic nanowires. Nature Photon. 2, 85-89 (2008).

13. Huang, Y., Duan, X. F., Wei, Q. Q. \& Lieber, C. M. Directed assembly of one-dimensional nanostructures into functional networks. Science 291, 630-633 (2001).

14. Ahn, J-H. et al. Heterogeneous three-dimensional electronics by use of printed semiconductor nanomaterials. Science 314, 1754-1757 (2006).

15. Park, W. I. et al. Controlled synthesis of millimeter-long silicon nanowires with uniform electronic properties. Nano Lett. 8, 3004-3009 (2008).

16. Sazio, P. J. A. et al. Microstructured optical fibers as high-pressure microfluidic reactors. Science 311, 1583-1586 (2006).

17. Suryavanshi, A. P., Hu, J. \& Yu, M. F. Meniscus-controlled continuous fabrication of arrays and rolls of extremely long micro- and nano-fibers. Adv. Mater. 20, 793-796 (2008).

18. Martensson, T. et al. Fabrication of individually seeded nanowire arrays by vapour-liquid-solid growth. Nanotechnology 14, 1255-1258 (2003).

19. McAlpine, M. C., Ahmad, H., Wang, D. W. \& Heath, J. R. Highly ordered nanowire arrays on plastic substrates for ultrasensitive flexible chemical sensors. Nature Mater. 6, 379-384 (2007).

20. Russell, P. Photonic crystal fibers. Science 299, 358-362 (2003).

21. Bayindir, M. et al. Metal-insulator-semiconductor optoelectronic fibres. Nature 431, 826-829 (2004).

22. Abouraddy, A. F. et al. Towards multimaterial multifunctional fibres that see, hear, sense and communicate. Nature Mater. 6, 336-347 (2007).

23. Bayindir, M. et al. Kilometer-long ordered nanophotonic devices by preform-to-fiber fabrication. IEEE J. Sel. Quant. Electron. 12, 1202-1213 (2006).

24. Yildirim, A., Vural, M., Yaman, M. \& Bayindir, M. Bio-inspired optoelectronic nose with nanostructured wavelength scalable hollow-core infrared fibers. Adv. Mater. 22, 1263 (2011).

25. Tong, L. M. et al. Subwavelength-diameter silica wires for low-loss optical wave guiding. Nature 426, 816-819 (2003).

26. Zhang, X. J., Ma, Z. Y., Yuan, Z. Y. \& Su, M. Mass-productions of vertically aligned extremely long metallic micro/nanowires using fiber drawing nanomanufacturing. Adv. Mater. 20, 1310-1314 (2008).

27. Deng, D. S. et al. Processing and properties of centimeter-long, in-fiber, crystalline-selenium filaments. Appl. Phys. Lett. 96, 023102 (2010).

28. Deng, D. S. et al. In-fiber semiconductor filament arrays. Nano Lett. 8, 4265-4269 (2008).

29. Donald, I. W. Production, properties and applications of microwire and related products. J. Mater. Science 22, 2661-2679 (1987).

30. Bayindir, M. et al. Thermal-sensing fiber devices by multimaterial codrawing. Adv. Mater. 18, 845-849 (2006).

31. Sun, C. L. et al. Fabrication and characterization of Ni/P(VDF-TrFE) nanoscaled coaxial cables. Appl. Phys. Lett. 90, 253107 (2007).

32. Tyagi, H. K. et al. Plasmon resonances on gold nanowires directly drawn in a step-index fiber. Opt. Lett 35, 2573-2575 (2010)

33. Danto, S. et al. Fibre field-effect device via in situ channel crystallization. Adv. Mat. 22, 4162-4166 (2010).

34. Keck, P. A. Photoconductivity in vacuum coated selenium films. J. Opt. Soc. Am. 42, 221-224 (1952).

35. Lee, S. H. et al. Highly-scalable nonvolatile and ultra-low power phase-change nanowire memory. Nature Nanotech. 2, 626-630 (2007).

36. Yu, B. et al. Chalcogenide-nanowire-based phase change memory. IEEE Trans. Nanotech. 7, 496-502 (2008).

37. Tian, B. et al. Coaxial silicon nanowires as solar cells and nanoelectronic power sources. Nature 449, 885-889 (2007).

38. Kelzenberg, M. D. et al. Enhanced absorption and carrier collection in Si wire arrays for photovoltaic applications. Nature Mater. 9, 239-244 (2010). 
39. Cao, L. et al. Semiconductor nanowire optical antenna solar absorbers. Nano Lett. 10, 439-445 (2010).

40. Grandidier, J., Callahan, D. M., Munday, J. N. \& Atwater, H. A. Light absorption enhancement in thin-film solar cells using whispering gallery modes in dielectric nanospheres. Adv. Mater. 23, 1272-1276 (2011).

41. Cao, L. et al. Engineering light absorption in semiconductor nanowire devices. Nature Mater. 8, 643-647 (2009).

42. Cao, L. et al. Tuning the color of silicon nanostructures. Nano Lett. 10, 2649-2654 (2010).

43. Vynck, K. et al. All-dielectric rod-type metamaterials at optical frequencies. Phys. Rev. Lett. 102, 133901 (2009).

44. Eggleton, B. J. et al. Chalcogenide photonics. Nature Photon. 5, 141-148 (2011).

45. Minardi, S. et al. Three-dimensional light bullets in arrays of waveguides. Phys. Rev. Lett. 105, 263901 (2010).

46. Egusa, S. et al. Multimaterial piezoelectric fibres. Nature Mater. 9, 643-648 (2010).

47. Chang, C. et al. Direct-write piezoelectric polymeric nanogenerator with high energy conversion efficiency. Nano Lett. 10, 726-731 (2010).

\section{Acknowledgements}

This work was partially supported by the State Planning Organization (DPT) and TUBITAK under project No 106G090. M.B. acknowledges support from the Turkish Academy of Sciences Distinguished Young Scientist Award (TUBA GEBIP).

\section{Author contributions}

M.Y. and M.B. designed and carried out research, analysed data and wrote the paper. M.K., T.K., M.Y., and M.B. carried out fabrication of nanowires and nanotubes. E.O. and O.A. made photoconduction measurements, E.O.O. and H.D. took SEM and transmission electron microscope micrographs and E.K. and M.B. drew schematic representations.

\section{Additional information}

The authors declare no competing financial interests. Supplementary information accompanies this paper on www.nature.com/naturematerials. Reprints and permissions information is available online at http://www.nature.com/reprints. Correspondence and requests for materials should be addressed to M.B. 


\section{ERRATUM}

\section{Arrays of indefinitely long uniform nanowires and nanotubes}

Mecit Yaman, Tural Khudiyev, Erol Ozgur, Mehmet Kanik, Ozan Aktas, Ekin O. Ozgur, Hakan Deniz, Enes Korkut and Mehmet Bayindir

Nature Materials 10, 494-501 (2011); published online 12 June 2011; corrected after print 19 July 2011.

In the version of this Letter previously published, the key for Fig. 5c was incorrect. This error has now been corrected in the HTML and PDF versions. 